\title{
DISTRIBUTION AND SOME CHARACTERISTICS OF CYTOCHROME P-450 IN THE KIDNEY
}

\author{
Hitoshi ENDOU \\ Department of Pharmacology, Faculty of Medicine \\ University of Tokyo, 7-3-1 Hongo, Bunkyo-ku, \\ Tokyo 113. Japan
}

\begin{abstract}
Intrarenal distribution of cytochrome $\mathrm{P}-450$ (P-450) was investigated with different segments of isolated single nephrons from rabbits and rats by using a new ultra-micro method of $\mathbf{P}-450$ determination. In both animals, $\mathrm{P}-450$ was localized only in the proximal tubule. Other segments such as the glomerulus, the loop of Henle, the distal tubule, and the collecting tubule possessed no $\mathrm{P}-450$ at all. Within the proximal tubule, the straight portion $\left(\mathrm{S}_{2}\right.$ and/or $S_{3}$ segments) revealed a higher specific content of $P-450$ than the convoluted one. An inducer of $P-450,3,4$-benzo(a)pyrene increased the $P-450$ of a definite portion (second $3 \mathrm{~mm}$ from the glomerulus) almost doubly in rabbits. In rats, both 3 -methylcholanthrene (3MC) and $48 \mathrm{hr}$ starvation (Fast) induced $P-450$, but only the later increased laurate- $\omega$-hydroxylation. $P-450$ in the proximal convoluted and straight tubules was induced separately by Fast and $3 \mathrm{MC}$, respectively. These results indicate that renal mixed function oxidase should be confined to the proximal tubule and that, like the liver, multiple forms of renal $\mathrm{P}-450$ should exist in the different proximal segments. Key words: Cytochrome P-450, isolated nephron segments, sodium phenobarbital, 3-methyl cholomthrene, 3, 4-benzo(a)pyrene,starvation.
\end{abstract}

\section{INTRODUCTION}

Numerous papers have reported on the hepatic $\mathrm{P}-450$ in the past 20 years. On the contrary, extrahepatic P-450s have been studied especially in comparison with the

This work was supported by Grant-in-Aid for special Project Research. Fundamental research on toxicological action.

連絡先：巨113 東京都文京区本郷 $7-3-1$ 東京大学医学部・薬理学 遠 藤 仁 
hepatic P-450 not only in their functions but also in their multiplicity (Ellin and Orrenius, 1975 ; Serabjit-Singh et al., 1979 ; Wolf et al., 1980 ; Franklin et al., 1980 ; Liem et al., 1980 ; Okita et al., 1981 ; Robertson et al., 1981; Dees et al., 1982 ; Hasumura et al., 1983). Recently, Guengerich's group (Guengerich and Mason, 1979 ; Kaminsky et al., 1979) has reported that the antibodies against $P-450$ s purified from phenobarbital (PB) and 3-methylcholanthrene (3MC) treated rat liver could cross-react with $\mathrm{PB}$ and $3 \mathrm{MC}$ induced $\mathrm{P}-450$ s from kidney microsomes, demonstrating that there should be common $P-450$ subunit(s) between liver and kidney microsomes. More recently, we could identify that the molecular subunit(s) of rat kidney $P-450$ were apparently more simple than those of the liver by one dimensional continuous gradient polyacrylamide gel electrophoresis (Hasumura et al., 1983).

Although mixed function oxidase activity in the kidney with the exception of fatty acid $\omega$-hydroxylation (Anders, 1980) is generally much lower than that of the liver, renal P-450 should be confined to a specific population of the kidney. Since anatomical structure of the kidney is very complicated and the basic morphological and functional units of the kidney are nephrons, microdissection of a single nephron and $P-450$ determination in each segment would shed light on the clear-cut investigation of renal $P$ 450 .

The purpose of this study is to demonstrate $P-450$ localization within a single nephron in rabbits and rats, and to show existence of $\mathrm{P}-450$ multiple forms induced by the treatment with several compounds. A part of this study has been reported elsewhere (Endou et al., 1982a; Endou, 1983).

\section{MATERIALS AND METHODS}

Biochemical Materials : Cóllagenase (type I), $\beta$-nicotinamide adenine dinucleotide phosphate (reduced form, NADPH), 3, 3', 5, 5', -tetramethylbenzidine (TMBZ) and 3,4benzo(a)pyrene (BP) were obtained from Sigma and $3 \mathrm{MC}$ was from Spectrum Chemical MFG. Corp. Sodium phenobarbital was kindly donated by Fujinaga Pharmaceutical Co. Ltd. Radioactive lauric acid, $\left[12^{-14} \mathrm{C}\right]$-laurate was supplied by Commissariat a L'Energie Atomique. Silica gel thin layer, Kieselgel 60, was obtained from Merck. All other reagents were of the highest grade commercially available.

Treatment of animals : Male New Zealand white rabbits weighing between 1.8 and 2.2 $\mathrm{kg}$ were used. The rabbits were given a single intraperitoneal injection of BP ( $20 \mathrm{mg} /$ kg) dissolved in corn oil; the controls received an equivalent volume of the corn oil. After $24 \mathrm{hr}$, the animals were sacrificed.

Male Sprague-Dawley rats weighing between 160 and $220 \mathrm{~g}$ were treated as described : $12 \mathrm{~h}$ fast ; $48 \mathrm{hr}$ fast ; daily intraperitoneal injection of $\mathrm{BP}(20 \mathrm{mg} / \mathrm{kg}), 3 \mathrm{MC}$ $(30 \mathrm{mg} / \mathrm{kg})$ dissolved in corn oil for 3 days, and PB $(80 \mathrm{mg} / \mathrm{kg})$ in saline for 3 days; and control (i. p. injection of corn oil or saline).

Preparation of microsomes: Microsomes were prepared from perfused kidneys. Homogenates in $0.25 \mathrm{M}$ sucrose were centrifuged at $10,000 \times \mathrm{g}$ for $10 \mathrm{~min}$. The supernatants were recentrifuged at $105,000 \times \mathrm{g}$ for $60 \mathrm{~min}$. The pellets (microsomes) were 
washed twice with $0.15 \mathrm{M} \mathrm{KCl}$ and suspended in $0.1 \mathrm{M}$ potassium phosphate buffer (pH 7.4) containing $1 \mathrm{mM}$ ED'TA and $20 \%$ glycerol.

Microdissection of nephron segments : Each nephron segment was dissected from perfused rabbit kidneys with $0.1 \%$ collagenase and rat kidneys with $0.1 \%$ collagenase and $0.1 \%$ hyaluronidase by the modified method (Endou et al., 1983) of Morel et al. (1978). Since $\mathbf{P}-450$ activities of the microdissected rat nephron segments decreased more rapidly than those of the rabbit, each segment from rats was used for $P-450$ assay within $2 \mathrm{hrs}$ after the enzyme treatment. Ten glomeruli and each 3 to $4 \mathrm{~mm}$ long nephron segment were used for $P-\mathbf{4 5 0}$ analysis and for protein measurements.

Analytical Procedures : Protein was measured according to Lowry et al. (1951).

The amount of $P-450$ was measured as described by Omura and Sato (1964) using a Hitachi 340 Dual Beam Recording Spectrophotometer.

Estimation of laurate- $\omega$-hydroxylation with renal microsomes prepared from treated rats was made by a modified method of Ichihara et al. (1969). Metabolites of lauric acid were separated on a thin-layer of silica gel 60 with ether-benzene-ethanol-formic acid $(250: 250: 10: 1$, by volume) as the solvent. The radioactivities of $\omega$-hydroxylated metabolites, identified with standards of authentic metabolites and laurate, were measured by liquid scintillation counting.

Micro-assay of $P-450$ (Endou, 1983) was based upon minimizing continuous gradient polyacrylamide gels (Ruechel et al., 1973) in which peroxidase activity of P-420 (Hrycay and O'Brien, 1971), a spectrally modified form of P-450 with sodium dodecyl sulfate (SDS), was stained with TMBZ and hydrogen peroxide (Thomas et al., 1976). Densitograms of stained gels in $7 \%$ acetic acid were made with a Joyce-LoebI MK III CS microdensitometer. Quantitative estimation of $P-450$ was carried out by measuring the peak height on the chart and comparing it with a standard line of peroxidase stained peak heights using partially purified $P-450$ prepared from rat kidney microsomes by the method of Imai and Sato (1974).

\section{RESULTS}

I. Rabbit Experiments.

Distribution of P-450 in kidney slices and effect of BP: Since renal $\mathrm{P}-450$ was distributed not only in the microsomes (Kato, 1966) but also in the mitochondria (Ghazarian et al., 1974), specific contents of $\mathrm{P}-450$ in the subcellular fractions from different slices were determined. As shown in Figure 1, renal microsomal $P-450$ was distributed in the cortex as well as in the outer medulla. Although no result is depicted in Figure 1, $\mathbf{P}-450$ was not detected in the inner medulla (also named papilla). It may be noteworthy that considerable amounts of $P-450$ were measured in the mitochondrial fractions $(10,000 \times g$ pellets) from both the cortex and the outer medulla. By the treatment with BP, only microsomal $\mathbf{P}-\mathbf{4 5 0}$ from the cortex was induced almost doubly, but not that from the outer medulla, suggesting that a BP-inducible P-450 subunit may be enriched in certain nephron segment(s) of the cortex. 


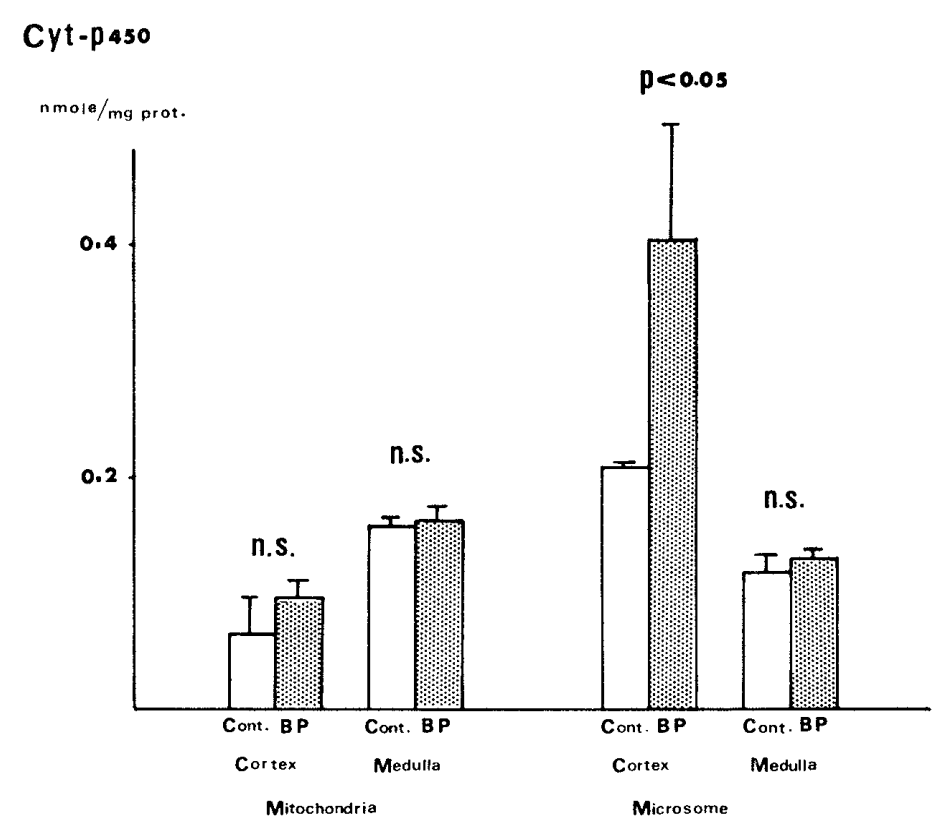

Fig. 1. Distribution of $\mathbf{P}-\mathbf{4 5 0}$ content in mitochondrial and microsomal fractions from control and BP treated rabbit slices. Column height and bars represent the mean $\pm S . D ., n=4$. The white column indicates the control and the grey one, $B P$ treatment. Since the papilla possessed no $P-450$ in both fractions, data are not depicted.

Intranephron distribution of P-450 : Peroxidase activities of hemeproteins containing $\mathrm{P}-420$, a modified form of $\mathrm{P}-450$ by SDS treatment, were stained after SDS gel electrophoresis. Under this condition only hemeproteins could be visualized, since the peroxidase activity of non-hemeproteins was inactivated in the presence of SDS. Figure 2 shows the intranephron distribution of $P-450$ in rabbits. It is striking to observe that only the proximal tubule contained $P-450$ and that other segments such as the glomerulus, the distal tubule, and the cortical collecting tubule possessed no $P-450$. Although results are not shown, no $\mathrm{P}-450$ could be detected in the loop of Henle. Within the proximal tubule, the $S_{2}$ segment, which consists of the late convoluted and early straight portions, revealed the highest specific contents of $\mathbf{P}-450$ per mm length as well as per $\mu$ g protein.

Increasing effect of BP on the intraproximal P-450 : According to the results depicted in Figure 1, it can be speculated that the $\mathbf{P}-450$ of certain nephron segment(s) within the cortex should be specifically induced. Since renal $P-450$ was distributed only in the proximal tubule (Figure 2), $\mathbf{P}-450$ contents along the single proximal tubule were estimated serially. As clearly demonstrated in Table $1, P-450$ of a definite portion, the second $3 \mathrm{~mm}$ segment, was increased significantly after the treatment with BP, indicating that the molecular subunit of $P-450$ induced by $B P$ may be enriched in this portion. Effect of BP on densitometric profiles of P-450 : Figure 3 represents typical densitograms of hemeproteins from control and BP treated $S_{2}$ segments of the proximal 


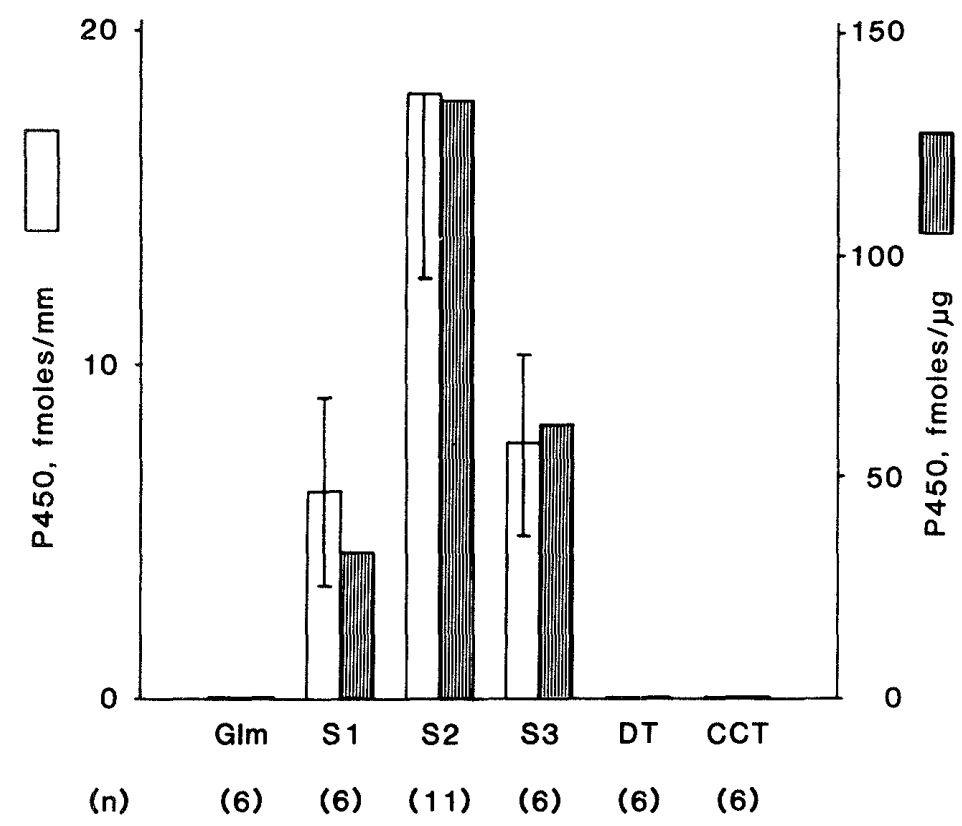

Fig. 2. Distribution of $P-450$ along a single nephron in rabbits. Each column and corresponding bar represent the mean $\pm S$. $D$. Numbers in parenthesis indicate number of samples assayed separately. Hatched column indicates specific content, fmole $/ \mu \mathrm{g}$ protein, which was calculated from P-450 content per mm length (value of white column) and protein amount of each nephron segment. Abbreviations of the nephron segments are; the glomerulus (Glm), segment 1 to 3 of the proximal tubule $\left(S_{1}-S_{3}\right)$, the distal tubule (DT), and the cortical collecting tubule (CCT).

tubules. At least 2 peaks of $P-450$ could be identified ; the main peak in the control and the predominant peak in the BP treated tubule. By the treatment with BP, a subpeak located at the anodic site in the control increased and became a predominant peak as clearly shown at the lower chart of Figure 3 . Since 4 to $40 \%$ continuous gradient polyacrylamide gels were used in this study, the molecular weight of the BP inducible peak should be larger than the main peak in the control.

II. Rat experiments.

Effects of several P-450 inducers on laurate- $\omega$-hydroxylation : Figure 4 represents specific $P-450$ contents and laurate- $\omega$-hydroxylation activities of rat kidney microsomes after fast for $12 \mathrm{hrs}$ and $48 \mathrm{hrs}$, and after the treatment with BP, 3MC, and PB. Fast induced a time-dependent increase of $P-450$ contents and laurate- $\omega$-hydroxylation. On the other hand, $\mathrm{BP}$ and $\mathbf{3 M C}$ induced $\mathrm{P}-\mathbf{4 5 0}$ but not laurate- $\omega$-hydroxylation. PB changed neither $\mathbf{P}-450$ nor laurate- $\omega$-hydroxylation. These results indicate that there should be at least two different $\mathrm{P}-450$ molecules in rat kidney microsomes : with and without increasing activity of laurate- $\omega$-hydroxylation.

Distribution of P-450 along a single nephron of rats : As can be observed in Figure 5, P-450 was localized only in the proximal tubule also in rats. Within the proximal 


\section{Hitoshi ENDOU}

Table 1. P-450 content in various segments of the proximal tubule from control and BP treated rabbits.

\begin{tabular}{lccc}
\hline & \multicolumn{2}{c}{ P-450, fmoles/ $\mu$ g protein } & \\
\cline { 2 - 3 } & Control & BP & P value \\
\hline First 3 mm from glomerulus & $50.9^{*}$ & 67.1 & NS \\
Second 3 mm from glomerulus & 49.0 & 103.9 & $<0.01$ \\
Third 3 mm from glomerulus & 93.0 & 135.5 & NS \\
Second 3 mm from terminal portion & 174.2 & 176.2 & NS \\
First 3 mm from terminal portion & 142.6 & 107.4 & NS \\
\hline
\end{tabular}

* All values are the means of two to four determinations.

NS represents no statistical significance.

EFFECT OF 3,4-BENZPYRENE ON HEMEPROTEIN PATTERN

OF STRAIGHT PROXIMAL TUBULE IN RABBIT

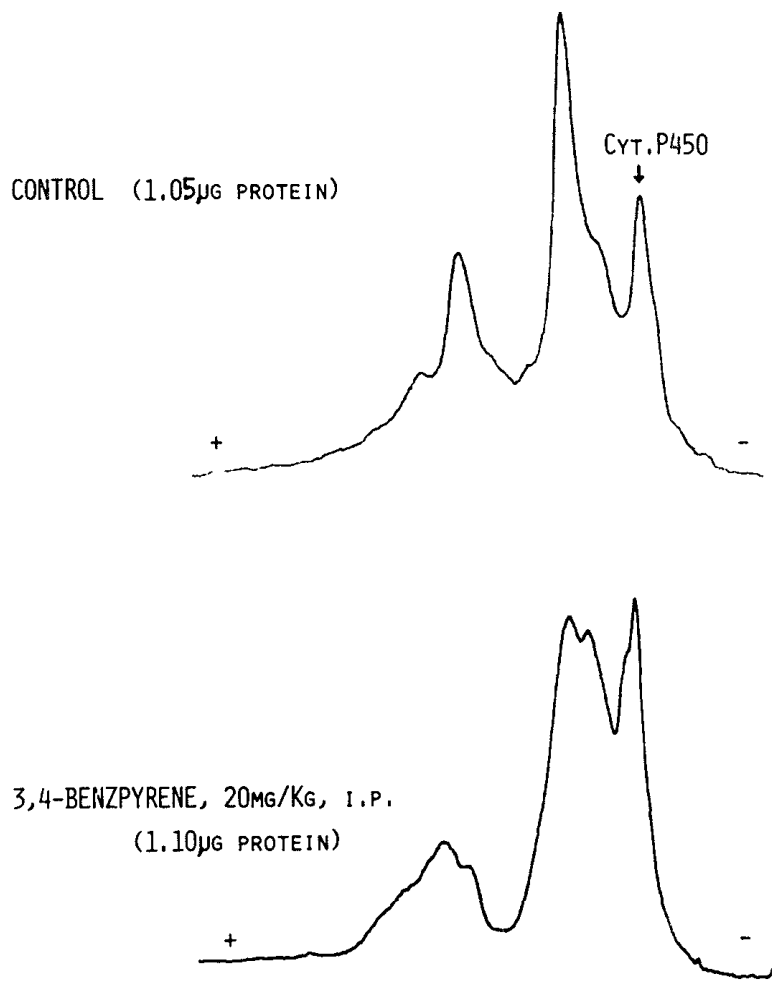

Fig. 3. Densitometric profiles $(620 \mathrm{~nm})$ of SDS-polyacrylamide gels stained with TMBZ and hydrogen dioxide after electrophoresis of the $S_{2}$ segments. In the control segment, a prominent peak with a slightly visible shoulder on the right side (higher molecular side) can be observed. After treatment with BP, the peak corresponding to the shoulder increased and became a dominant peak as is clearly shown at the bottom. 


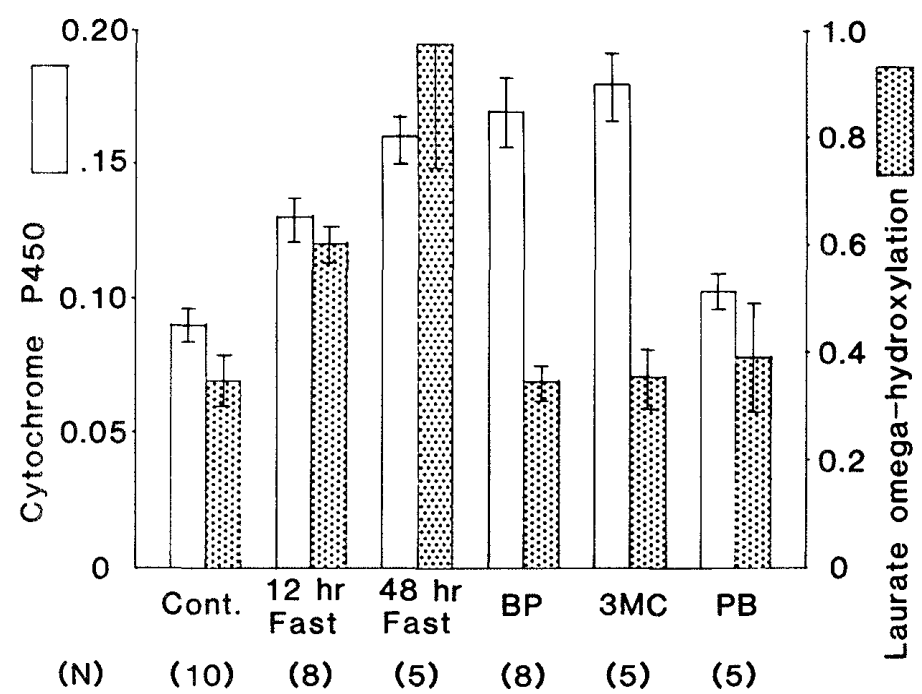

Fig. 4. Effects of inducers on microsomal $P-450$ and laurate $\omega$-hydroxylation in rats. White and hatched columns and bars represent the means \pm S. D.; P-450, nmoles/mg ; laurate $\omega$-hydroxylation, nmoles $/ \mathrm{mg} / 10 \mathrm{~min}$.

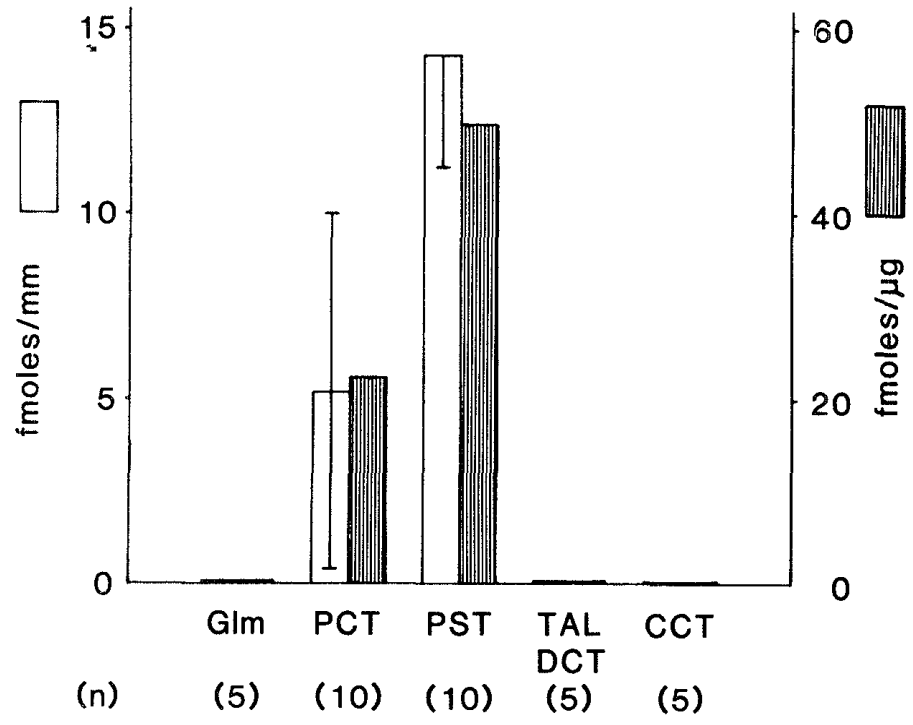

Fig. 5. Distribution of $P-450$ along a single nephron in rats. Each column and corresponding bar represent the mean $\pm S$. D. The abbreviations are the same as stated in Fig. 2, except the proximal convoluted tubule (PCT), the proximal straight tubule (PST), the thick ascending limb of Henle's loop (TAL), and the distal convoluted tubule (DCT). 

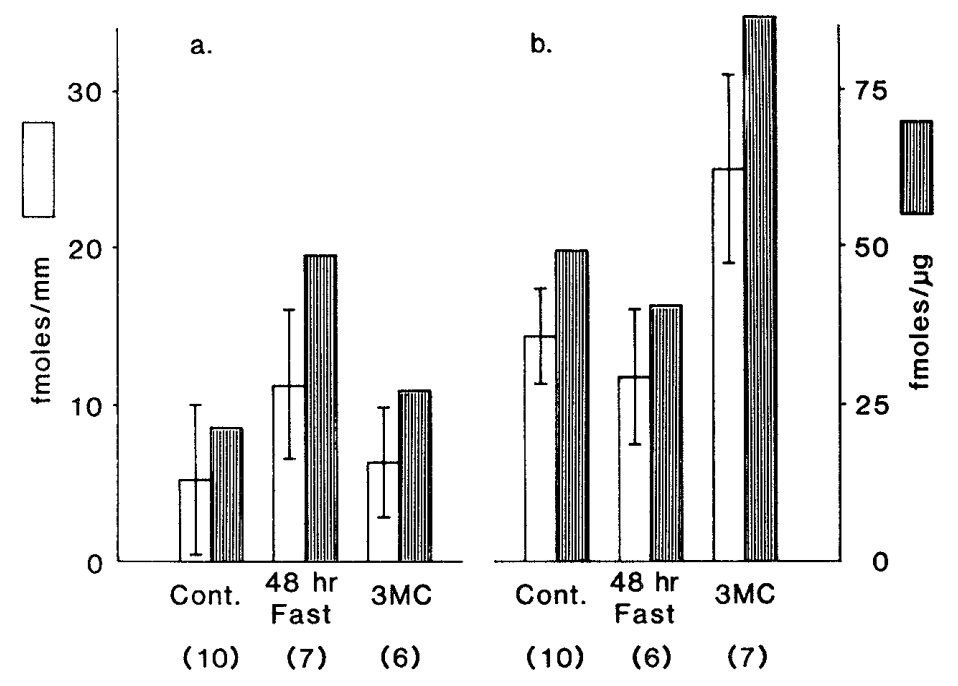

Fig. 6. Effects of 48-hour fast and $3 \mathrm{MC}$ on distributional patterns of $\mathrm{P}-450$ in $\mathrm{PCT}(\mathrm{a})$ and PST(b).

tubule, the straight tubule (PST) contained a higher specific P-450 content than the convoluted one (PCT). Although classification of nephron segments used in rat experiments is not the same as that in rabbits, these resullts (Figure 5) are fully consistent with those in rabbits (Figure 2), suggesting that there is no qualitative species difference in intranephron distribution of $P-450$ between rats and rabbits.

Distributional heterogeneity of P-450 multiple forms within the proximal tubule : Since an apparent discrepancy could be observed between $P-450$ contents and laurate- $\omega$ -hydroxylation activities induced by fast and chemicals such as BP and 3MC (Figure 4), changes of $\mathrm{P}-450$ contents in PCT and PST were determined by 48-hour fast and 3MC. Figure 6a shows changes in $\mathrm{P}-450$ contents of PCT from rats of control, $48 \mathrm{hr}$ fast, and 3MC treatment. Fast for 48 hrs significantly increased $P-450$ contents of PCT per mm length and per $\mu \mathrm{g}$ protein, whereas $3 \mathrm{MC}$ did not change $\mathrm{P}-450$ content in PCT. On the other hand, effects of fast and $3 \mathrm{MC}$ on P-450 contents of PST were the reverse (Figure 6b). Treatment of rats with $3 \mathrm{MC}$ caused $\mathrm{P}-450$ in PST to increase significantly. Fast for 48 hrs, however, did not affect $P-450$ contents in PST. These results suggest that two forms of $\mathrm{P}-\mathbf{4 5 0}$ may be distributed differently from PCT to PST in rats.

\section{DISCUSSION}

Intrarenal distribution of P-450: In 1966, Kato first reported the presence of $\mathrm{P}-450$ in rat kidney microsomes. Because of a lower specific content of $P-450$ in the kidney than the liver, less attention might have been previously paid to renal $\mathbf{P}-450$. A cortical -papillary gradient (highest in the cortex lowest in the papilla) for mixed function oxidase activities and $P-450$ contents has been reported by Zenser et al. (1978). Figure 
1 in this paper confirms this evidence. From the fact that rats treated with 2, 3, 7, 8 -tetrachlorodibenzo-p-dioxin (TCDD), an inducer of microsomal $P-450$, have increased renal $P-450$ and proliferation of the smooth endoplasmic reticulum in the $S_{3}$ cells of the proximal tubule (Fowler et al., 1977), intranephron distribution of P-450 could be speculated in the $S_{3}$ segment of the proximal tubule. More direct evidence, in which an antibody against purified microsomal $P-450$ from pig kidney cortex could bind to the proximal tubule cells (Masters et al., 1981), suggests that renal P-450 may be distributed in the proximal tubule. This qualitative finding is supported by the quantitative assays of $\mathbf{P}-\mathbf{4 5 0}$ with microdissected nephron segments in this paper (Figure 2 and 5).

Relationship between P-450 distribution and nephron functions : Renal P-450 is distributed not only in the microsomes (Kato, 1966), but also in the mitochondria (Ghazarian et al., 1974). Each P-450 plays important roles in drug metabolism (Anders, 1980), w-hydroxylation of medium-chain fatty acids (Ellin and Orrenius, 1975), and 25(OH)vitamin $D_{3}-1 \alpha$-hydroxylation (Ghazarian et al., 1974). In the kidney, mitochondrial $P-450$ is distributed in a fashion similar to the microsomal one within different slices (Figure 1) and proximal tubules (Endou, 1983). It may be interesting, therefore, to compare $\mathrm{P}-\mathbf{4 5 0}$ distribution in nephron segments (Figure 2 and 5) with intranephron distribution of $25(\mathrm{OH})$ vitamin $\mathrm{D}_{3}-1 \alpha$-hydroxylase activity. In rachitic chickens, $1 \alpha$-hydroxylase activity could be detected in PCT and the thick loop cortex (Brunette et al., 1978). Although the later portion is difficult to compare with the mammalian nephron because of differences in anatomical structure of avian nephrons, a part of the nephron, PCT in chickens, is consistent with the $P-450$ containing segment in this study (Figure 2 and 4). In rabbit fetal kidney, we could demonstrate that $1 \alpha$ -hydroxylase activity has been found only in the proximal tubule, with PST showing higher activity (Akiba et al., 1980). This finding is quite consistent with the present results of $\mathbb{P}-450$ localization in a single nephron. In vitamin D-deficient rats, PCT has been found to produce $1 \alpha, 25(\mathrm{OH})_{2}$ vitamin $\mathrm{D}_{3}$ more effectively than PST (Kawashima et al., 1981a). The discrepancy between higher $1 \alpha$-hydroxylase activity with lower amount of $\mathrm{P}-450$ in PCT in rats, and lower hydroxylase with higher $\mathrm{P}-450$ in PST has been explained by high blood level of calcitonin in the fetus which selectively stimulates $1 \alpha$-hydroxylase in PST (Kawashima et al., 1981b).

Multiple forms of renal P-450: The mitochondrial P-450 of the kidney has been shown to be distinct from the microsomal one (Ghazarian et al., 1974). As studied intensively with liver microsomal P-450(s) (Lu and West, 1980), multiple forms of microsomal P-450 in rabbit kidneys have been reported (Armbrecht et al., 1979; Liem et al., 1980 ; Kusunose et al., 1981; Ogita et al., 1982 ; Ogita et al., 1983). Two forms of P-450, of which molecular weights are 54,500 and 58,900 (Armbrecht et al., 1970), were detected. Similar results were found by Liem et al. (1980) and Ogita et al. (1982). Figure 3 in this paper also supports these findings. The result shown in Figure 3, in which treatment of rabbits with BP increased the higher molecular $P-450$ from the $S_{2}$ segment, is consistent with the clear evidence of $P-448$ enabling BP to catalyze (Ogita et al., 1982). Multiple forms of rat kidney microsomal $P-450$ could be purified, because the results in 


\section{Hitoshi ENDOU}

this study (Figure 4 and 6 ) suggest this possibility.

It may be more interesting to know that individual segments along the proximal tubule may contain different form(s) of $\mathrm{P}-450$ and specify distinct metabolic functions in each segment. Further study should be carried out on drug metabolism and $\omega-$ and/or ( $\omega-1)$-hydroxylation of endogenous active compounds such as prostaglandins and hormones using microdissected nephron segments and isolated kidney cells. We recently reported a trial for sorting isolated kidney cells in pure populations by a flow cytometer (Endou et al., 1982b).

\section{ACKNOWLEDGEMENTS}

The author thanks Miss N. Todoroki for her expert secretarial assistance. This study was supported in part by grants from the Japanese Ministry of Education, Science and Culture (No. 56122018 and 57570069), the Foundation for the Promotion of Research on Medicinal Resources, and Mitsukoshi Prize of Medicine 1982.

\section{REFERENCES}

Akiba, T., Endou, H., Koseki, C., Sakai, F., Horiuchi, N. and Suda, T. (1980) : Localization of 25-hydroxyvitamin $D_{3}-1 \alpha$-hydroxylase activity in the mammalian kidney. Biochem. Biophys. Res. Commun., 94, 313-318.

Armbrecht, H. J., Birnabum, L. S., Zenser, T. V., Mattammal, M. B. and Davis, B. B. (1979) : Renal cytochrome $P-450$ 's - electrphoretic and electron paramagnetic resonance studies. Arch. Biochem. Biophys., 197, 277-284.

Anders, M. W. (1980) : Metabolism of drugs by the kidney. Kidney Int., 18, 636-647.

Brunette, M. G., Chan, M., Ferriere, C. and Roberts, K. D. (1978) : Site of 1, 25(OH) $)_{2}$ vitamin $D_{3}$ synthesis in the kidney. Nature, 279, 287-289.

Dees, J. H., Masters, B. B. S., Muller-Eberhard, V. and Johnson, E. F. (1982) : Effect of 2, 3, 7, 8-tetrachloro-dibenzo-p-dioxin and phenobarbital on the occurence and distribution of four cy tochrome $\mathbf{P}-450$ isozymes in rabbit kidney, lung, and liver. Cancer Res., 42, 14231432.

Ellin,A. and Orrenius, S. (1975) : Fatty acid hydroxylation in rat kidney cortex microsomes. Molecular \& Cellular Biochem., 8, 69-79.

Endou, H., Koseki, C., Hasumura, S., Kakuno, K., Hojo, K. and Sakai, F. (1982a) : Renal cytochrome $\mathrm{P}-450$; its localization along a single nephron and its induction. In Biochemistry of kidney functions (F. Morel, ed.), pp. 319-327. Elsevier Biomedical Press Amsterdam.

Endou, H., Koseki, C., Kimura, K., Yokokura, Y., Fukuda, S. and Sakai, F. (1982b) : Use of a flow cytometer for the separation of isolated kidney cells. In Biochemistry of kidney functions (F. Morel, ed.), pp. 69-78. Elsevier Biomedical Press, Amsterdam.

Endou, H. (1983) : Cytochrome P-450 monooxygenase system in the rabbit kidney: Its intranephron localization and its induction. Japan. J. Pharmacol., 33, 423-433.

Endou, H., Kimura, K. and Koseki, C. (1983) : Methods for metabolic investigation of isolated nephron segments. Metabolism and Disease, 20, 479-488. (in Japanese) 
Franklin, K. R., Wold, V. R., Serabjit-Singh, C. and Philpot, R. M. (1980) : Quantitation of two forms of pulmonary cytochrome P-450 in microsomes, using substrate specificities. Mol. Pharmacol., 17, 415-420.

Fowler; B. A., Hook, G. E. R. and Lucier, G. W. (1977) : Tetrachlorodibenzo-p-dioxin induction of renal microsomal enzyme systems : Ultrastructural effects on pars recta $\left(\mathrm{S}_{3}\right)$ proximal tubule cells of the rat kidney. J. Pharmacol. Exp. Ther., 203, 712-721.

Ghazarian, J. G., Jefcoate, C. R., Knutson, T. C., Orme-Johnson, W. H. and DeLuca, H. F. (1974) : Mitochondrial cytochrome P-450. A component of chick kidney 25-hydroxycholecalciferol-1 $\alpha$-hydroxylase. J. Biol. Chem., 249, 3026-3033.

Hasumura, S., Endou, H., Kakuno, K., Hojo., K. and Sakai, F. (1983) : Effects of starvation on microsomal cytochrome $\mathbf{P}-450$ and laurate-c-hydroxylation of rat kidney and liver. Japan. J. Pharmacol. in press.

Hrycay, E. G. and O'Brien, P. J. (1971) : The peroxidase nature of cytochrome P-420 utilizing a lipid peroxide substrate. Arch. Biochem. Biophys., 147, 28-35.

Ichihara, K., Kusunose, E. and Kusunose, M. (1969) : Some properties and distribution of the $\omega-$ hydroxylation system of medium chain fatty acids. Biochim. Biophys. Acta, 176, 704-712.

Imai, Y. and Sato, R. (1974) : An affinity column method for partial purification of cytochrome P-450 from phenobarbital-induced rabbit liver microsomes. J. Biochem., 75, 689-697.

Kaminsky, L. S., Fasco, M. J. and Guengerich, F. P. (1979) : Comparison of different forms of liver, kidney, and lung microsomal cytochrome $P-450$ by immunological inhibition of regio - and stereoselective metabolism of warfarin. J. Biol. Chem., 254, 9657-9662.

Kato, R. (1966) : Possible role of $\mathrm{P}-450$ in the oxidation of drugs in liver microsomes. $J$. Biochem., 59, 574-583.

Kawashima, H., Torikai, S. and Kurokawa, K. (1981a) : Localization of 25-hydroxyvitamin $\mathrm{D}_{3}$ $1 \alpha$-hydroxylase and 24 -hydroxylase along the rat nephron. Proc. Natl. Acad.Sci. U.S. A., 78, 1199-1203.

Kawashima, H., Torikai, S. and Kurokawa, K. (1981b) : Calcitonin selectively stimulates 25hydroxyvitamin $D_{3}-1 \alpha$-hydroxylase in proximal straight tubule of rat kidney. Nature, 291, 327-329.

Kusunose, E., Kaku, M., Nariyama, M., Kusunose, M., Ichihara, K., Funae, Y, and Kotake, A. N. (1981): High-performance liquid chromatography of cytochrome $\mathbf{P}-450$ from rabbit liver, kidney cortex and intestinal mucosa microsomes. Biochem. Int., 3, 339-406.

Liem, H. H., Mullen-Eberhard, V. and Johnson, E. F. (1980) : Differential induction by 2, 3, 7, 8, tetrachloro-dibenzo-p-dioxin of multiple forms of rabbit microsomal cytochrome P-450: Evidence for tissue specificity. Mol. Pharmacol., 18, 565-570.

Lowry, P. G., Rosenbrough, N. J., Farr, A. L. and Randall, R. J. (1951) : Protein measurement with the folin phenol reagent. J. Biol. Chem., 193, 265-275.

Lu, A. Y. H. and West, S. B. (1980): Multiplicity of mammalian microsomal cytochromes P-450. Pharmacol. Rev., 31, 277-295.

Masters, B. S. S., Okita, R. T., Dees, J. H., Yasukochi, Y., Parkhill, L. K., Theoharides, A. D. and Kupfer, D. (1981) : Biochemical characterization and morphological localization of a cytochrome $\mathbf{P}-450$ in pig kidney cortex. Abstracts Fifth International Symposium on Microsomes and Drug Oxidations. Tokyo, p. 48.

Morel, F., Chabardes, D. and Imbert-Teboul, M. (1978) : Methodology for enzymatic studies of 


\section{Hitoshi ENDOU}

isolated tubular segments : Adenylate cyclase. In Methods in Pharmacology (M. Martinez-Maldonado, ed.), pp. 297-323. Plenum Press, New York and London.

Ogita, K., Kusunose, E., Ichihara, K. and Kusunose, M. (1982) : Multiple forms of cytochrome $\mathbf{P - 4 5 0}$ in kidney cortex microsomes of rabbits treated with 3 -methyhcholanthrene. $J$. Biochem., 92, $921-928$.

Ogita, K., Knsunose, E., Yamamoto, S., Ichihara, K. and Kusunose, M. (1983) : Multiple forms of cytochrome $P-450$ from kidney cortex microsomes of rabbits treated with phenobarbital. Biochem. Int., 6, 191-198.

Okita, T. R., Parkhill, L. K., Yasukochi, Y. and Masters, B. S. S. (1981) : The $\omega-$ and ( $\omega-1)-$ hydroxylase activities of prostaglandins $A_{1}$ and $E_{1}$ and lauric acid by pig kidney microsomes and a purified kidney cytochrome $P-450$. J. Biol. Chem., 256, 5961-5964.

Omura, T. and Sato, R. (1964) : The carbon monoxide-binding pigment of liver microsomes. J. Biol. Chem., 239, 2370-2378.

Robertson, I. G.C., Philpot, R. M., Zeiger, E. and Wolf, C. R. (1981) : Specificity of rabbit pulmonary cytochrome $P-450$ isozymes in the activation of several aromatic amines and aflatoxin $B_{1}$. Mol. Pharmacol., 20, 662-668.

Ruechel, R., Mesecke, S., Wolfrum, D. I. and Neuhoff, V. (1973): Mikroelektrophorese an kontinuierlichen Polyacrylamid Gradienten Gelen. I. Herstellung und Eigenschaften von Gelgradienten in Kapillaren; ihre Anwendung zur Proteinfraktionierung und Moleklargewichtsbestimmung. Hoppe-Seyler's Z. Physiol. Chem., 354, 1351-1368.

Serabjit-Singh, C. J., Wolf, C. R. and Philpot, R. M. (1979) : Cytochrome P-450 : Localization in rabbit lung. Science, 207, 1469-1470.

Thomas, P.E., Ryan, D. and Levin, W. (1976) : An improved staining procedure for the detection of the peroxidase activity of cytochrome $\mathrm{P}-450$ on sodium dodecyl sulfate polyacrylamide gels. Anal. Biochem., 75, 168-176.

Wolf, C. R., Slaughter, S. R., Marchiniszyn, J. P. and Philpot, R. M. (1980) : Purification and structural comparison of pulmonary and hepatic cytochrome $P-450$ from rabbits. Biochim. Biophys. Acta, 624, 409-419.

Zenser, T. V., Mattammal, M. B. and Davis, B.B. (1978): Differential distribution of the mixed-function oxidase activities in rabbit kidney. J. Pharmacol. Exp. Ther., 207, 719 $-725$. 